\title{
Attractors for 2D-Navier-Stokes equations with delays on some unbounded domains
}

\author{
Pedro Marín-Rubio ${ }^{\text {a,*}}$, José Real $^{\text {a }}$ \\ ${ }^{a}$ Dpto. de Ecuaciones Diferenciales y Análisis Numérico, Universidad de Sevilla, \\ Apdo. de Correos 1160, 41080 Sevilla, Spain
}

\begin{abstract}
We prove the existence of tempered and non-tempered pullback attractors for two dimensional Navier-Stokes equations on unbounded domains satisfying Poincaré inequality, for the case in which a forcing term involving memory effects appears. Our proof uses an energy method and is valid for the autonomous and non-autonomous cases.
\end{abstract}

Key words: Navier-Stokes equations, delays terms, unbounded domains, asymptotic compactness, attractors.

\section{Introduction}

Navier-Stokes equations have received very much attention over the last decades due to their importance in the understanding of fluid motion and turbulence. No doubt at all, the asymptotic behaviour of general dynamical systems, and particularly for the NS equations, is an interesting and challenging problem, since it can provide useful information on the future evolution of the system (see $[5-7,9,11,18,19]$ amongst others).

Very recently, in [2-4] there started the research involving Navier-Stokes models in which the forcing term contains some hereditary features. These situations may appear, for instance, when we want to control the system by applying a force which takes into account not only the present state of the system but the history of the solutions.

\footnotetext{
* Corresponding author

Email addresses: pmr@us.es (Pedro Marín-Rubio), jreal@us.es (José Real).
} 
After establishing some sufficient conditions ensuring the exponential behaviour of solutions to a 2DNavier-Stokes delay model (essentially when the viscosity is large, and every solution converges towards the unique stationary solution, cf. [3] ), the attractor is the next interesting concept to study under more relaxed assumptions.

More precisely, when the viscosity is small one can expect something similar to what happens in the non-delay framework, i.e., the existence of a compact invariant attracting set (a global attractor for the associated semigroup). But on this framework, it is necessary to be careful with the analysis, concretely with the phase space in order to consider the associated semigroup. In fact, the dynamical system needs to be defined in a phase space of trajectories.

Although for some particular cases, the resulting abstract equation becomes autonomous (e.g. for constant delays) and the standard techniques for autonomous dynamical systems can be adapted to solve the problem, most cases need a non-autonomous model to describe the system and, consequently, a non-autonomous technique is necessary to handle the problem. Concretely, in [4] the authors study the existence of pullback attractors for non-autonomous systems (although other options are also valid, as kernel sections or skewproduct formalism).

However, a crucial point in this last work is that the process is compact (the boundedness of the domain plays a crucial role for compact Sobolev embedding). Autonomous and non-autonomous problems without the compactness property and in a non-delay framework have been treated, for instance, in $[14,13,1]$. Existence, uniqueness, and exponential stability of the stationary solution in the case of some unbounded domains and with presence of delays was studied in [8].

The purpose of this paper is to extend the above results on attractors to the case where the domain $\Omega$ is not necessarily bounded, under more relaxed conditions than in [8], by the way of proving an asymptotic compactness property. Namely, we extend some of the results in [4] to the case of unbounded domains satisfying Poincaré inequality. In our proof there is no need of regularity of the boundary of $\Omega$. A crucial point is that we make use of the tempered framework, which also allows us to ensure existence and some nice properties on the attractor for the non-tempered case. Both situations have an immediate translation into the autonomous case.

The contents of the rest of the paper are as follows. In Section 2 we will pose the statement of the problem. Section 3 is devoted to recall some useful results on dynamical systems and attractor theory, pointing out specially the benefit of tempered framework. In Section 4 the construction of the process and the main results are given. Namely, these are continuity properties of the process, 
existence of an absorbing set, and asymptotic compactness in a suitable universe, to conclude with the existence of tempered and non-tempered pullback attractors.

\section{Statement of the problem}

Let $\Omega \subset \mathbb{R}^{2}$ be an open set with boundary $\Gamma$. We do not suppose that $\Gamma$ is regular, and $\Omega$ is not necessarily bounded, but satisfies a Poincaré inequality, i.e., there exists $\lambda_{1}>0$ such that

$$
\int_{\Omega}|\phi|^{2} \mathrm{~d} x \leq \frac{1}{\lambda_{1}} \int_{\Omega}|\nabla \phi|^{2} \mathrm{~d} x, \quad \text { for all } \phi \in H_{0}^{1}(\Omega) .
$$

We consider the following functional Navier-Stokes problem:

$$
\left\{\begin{array}{l}
\frac{\partial u}{\partial t}-\nu \Delta u+\sum_{i=1}^{2} u_{i} \frac{\partial u}{\partial x_{i}}=f(t)-\nabla p+g\left(t, u_{t}\right) \quad \text { in }(\tau,+\infty) \times \Omega, \\
\operatorname{div} u=0 \quad \text { in }(\tau, T) \times \Omega, \\
u=0 \text { on }(\tau,+\infty) \times \Gamma \\
u(\tau, x)=u^{0}(x), \quad x \in \Omega, \\
u(t, x)=\phi(t-\tau, x), \quad t \in(\tau-h, \tau) \quad x \in \Omega,
\end{array}\right.
$$

where $\nu>0$ is the kinematic viscosity, $u$ is the velocity field of the fluid, $p$ the pressure, $\tau \in \mathbb{R}$ the initial time, $u_{0}$ the initial velocity field, $f$ a nondelayed external force field, $g$ another external force with some hereditary characteristics and $\phi$ the initial datum in the interval of time $(-h, 0)$, where $h$ is a fixed positive number. We denote by $u_{t}$ the function defined on $(-h, 0)$ by the relation $u_{t}(s)=u(t+s), s \in(-h, 0)$.

To set our problem in the abstract framework, we consider the usual abstract spaces:

$$
\mathcal{V}=\left\{u \in\left(C_{0}^{\infty}(\Omega)\right)^{2}: \operatorname{div} u=0\right\}
$$

$H=$ the closure of $\mathcal{V}$ in $\left(L^{2}(\Omega)\right)^{2}$ with the norm $|\cdot|$, and inner product $(\cdot, \cdot)$ where for $u, v \in\left(L^{2}(\Omega)\right)^{2}$,

$$
(u, v)=\sum_{j=1}^{2} \int_{\Omega} u_{j}(x) v_{j}(x) \mathrm{d} x,
$$

$V=$ the closure of $\mathcal{V}$ in $\left(H_{0}^{1}(\Omega)\right)^{2}$ with the norm $\|\cdot\|$ associated to the inner 
product $((\cdot, \cdot))$, where for $u, v \in\left(H_{0}^{1}(\Omega)\right)^{2}$,

$$
((u, v))=\sum_{i, j=1}^{2} \int_{\Omega} \frac{\partial u_{j}}{\partial x_{i}} \frac{\partial v_{j}}{\partial x_{i}} \mathrm{~d} x .
$$

It follows that $V \subset H \equiv H^{\prime} \subset V^{\prime}$, where the injections are dense and continuous. We will use $\|\cdot\|_{*}$ for the norm in $V^{\prime}$ and $\langle\cdot, \cdot\rangle$ for the duality $\left\langle V^{\prime}, V\right\rangle$. Now we define $A: V \rightarrow V^{\prime}$ by $\langle A u, v\rangle=((u, v))$, and the trilinear form $b$ on $V \times V \times V$ by

$$
b(u, v, w)=\sum_{i, j=1}^{2} \int_{\Omega} u_{i} \frac{\partial v_{j}}{\partial x_{i}} w_{j} \mathrm{~d} x \quad u, v, w \in V .
$$

Let us denote $B: V \times V \rightarrow V^{\prime}$ the operator given by $\langle B(u, v), w\rangle=b(u, v, w)$, for all $u, v, w \in V$, and $B(u)=B(u, u)$.

We remember that

$$
b(u, v, w)=-b(u, w, v), \text { for all } u, v, w \in V,
$$

and in particular

$$
b(u, v, v)=0, \text { for all } u, v \in V .
$$

The following inequality holds (see [12]):

$$
|b(u, v, u)| \leq 2^{-1 / 2}|u|\|u\|\|v\|, \text { for all } u, v \in V .
$$

We denote $C_{H}=C^{0}([-h, 0] ; H)$, and $L_{X}^{2}=L^{2}(-h, 0 ; X)$ for $X=H, V, V^{\prime}$.

Now, we establish suitable hypotheses on the term containing the delay. Let $g: \mathbb{R} \times C_{H} \rightarrow\left(L^{2}(\Omega)\right)^{2}$ satisfy the following assumptions:

(I) for all $\xi \in C_{H}$, the function $t \in \mathbb{R} \rightarrow g(t, \xi) \in\left(L^{2}(\Omega)\right)^{2}$ is measurable,

(II) $g(t, 0)=0$, for all $t \in \mathbb{R}$,

(III) there exists $L_{g}>0$ such that for all $t \in \mathbb{R}$, and for all $\xi, \eta \in C_{H}$,

$$
|g(t, \xi)-g(t, \eta)| \leq L_{g}\|\xi-\eta\|_{C_{H}}
$$

(IV) there exists $C_{g}>0$ such that for all $\tau \leq t$, and for all $u, v \in C^{0}([\tau-h, t] ; H)$,

$$
\int_{\tau}^{t}\left|g\left(s, u_{s}\right)-g\left(s, v_{s}\right)\right|^{2} \mathrm{~d} s \leq C_{g}^{2} \int_{\tau-h}^{t}|u(s)-v(s)|^{2} \mathrm{~d} s .
$$

Observe that (I)-(III) imply that given $u \in C^{0}([\tau-h, T] ; H)$, the function $g_{u}: t \in[\tau, T] \rightarrow\left(L^{2}(\Omega)\right)^{2}$ defined by $g_{u}(t)=g\left(t, u_{t}\right) \forall t \in[\tau, T]$, is measurable 
and, in fact, belongs to $L^{\infty}\left(\tau, T ;\left(L^{2}(\Omega)\right)^{2}\right)$. Then, thanks to (IV), the mapping

$$
\mathcal{G}: u \in C^{0}([\tau-h, T] ; H) \rightarrow g_{u} \in L^{2}\left(\tau, T ;\left(L^{2}(\Omega)\right)^{2}\right)
$$

has a unique extension to a mapping $\widetilde{\mathcal{G}}$ which is uniformly continuous from $L^{2}(\tau-h, T ; H)$ into $L^{2}\left(\tau, T ;\left(L^{2}(\Omega)\right)^{2}\right)$. From now on, we will denote $g\left(t, u_{t}\right)=$ $\widetilde{\mathcal{G}}(u)(t)$ for each $u \in L^{2}(\tau-h, T ; H)$, and thus property (IV) will also hold for all $u, v \in L^{2}(\tau-h, T ; H)$.

We will suppose that $g$ also satisfies:

(V) if $\mathcal{O}$ is a bounded open subset of $\Omega$, and the sequence $\left\{v^{m}\right\} \subset L^{2}(\tau-h, T ; H)$ converges to $v$ strongly in $L^{2}\left(\tau-h, T ;\left(L^{2}(\mathcal{O})\right)^{2}\right)$, with $v \in L^{2}(\tau-h, T ; H)$, then $g\left(\cdot, v^{m}\right)$ converges weakly to $g(\cdot, v$. $)$ in $L^{2}\left(\tau, T ;\left(L^{2}(\mathcal{O})\right)^{2}\right)$.

Let us assume now that $u_{0} \in H, \phi \in L_{H}^{2}, f \in L_{\text {loc }}^{2}\left(\mathbb{R} ; V^{\prime}\right)$, and $g: \mathbb{R} \times C_{H} \rightarrow$ $\left(L^{2}(\Omega)\right)^{2}$ satisfies hypotheses (I)-(V). Examples of such operators $g$ can be found in $[8$, Sec.3].

For each $\tau \in \mathbb{R}$ we consider the problem:

$$
\left\{\begin{array}{l}
\text { To find } u \in L^{2}(\tau-h, T ; H) \cap L^{2}(\tau, T ; V) \cap L^{\infty}(\tau, T ; H) \forall T>\tau, \\
\frac{\mathrm{d}}{\mathrm{d} t} u(t)+\nu A u(t)+B(u(t))=f(t)+g\left(t, u_{t}\right) \text { in } \mathcal{D}^{\prime}\left(\tau,+\infty ; V^{\prime}\right), \\
u(0)=u^{0}, u(t)=\phi(t-\tau), \text { for a.a. } t \in(\tau-h, \tau) .
\end{array}\right.
$$

The following result can be proved in the same manner as the existence and uniqueness theorem proved in [8]:

Theorem 1 Let $u^{0} \in H, \phi \in L^{2}(-h, 0 ; H), f \in L_{\text {loc }}^{2}\left(\mathbb{R} ; V^{\prime}\right)$, and assume that $g: \mathbb{R} \times C_{H} \rightarrow\left(L^{2}(\Omega)\right)^{2}$ satisfies hypotheses (I)-(V). Then, for each $\tau \in \mathbb{R}$, there exists a unique solution of $(2)$, denoted $u\left(t, \tau, u^{0}, \phi\right)$, which, in addition, belongs to $C^{0}([\tau,+\infty) ; H)$.

\section{Preliminaries on attractors}

Our goal is to study the asymptotic behaviour for solutions of the problem (2). The possibly unbounded character of the domain and the lack of regularity of its boundary do not allow us to apply directly the techniques of compact semiflows and semiprocesses for this problem. Here we recall briefly some of the essential concepts and results in order to state existence of attractor for the dynamical system associated to $(2)$ (see also $[1,7,15]$ ). 
We make the exposition for non-autonomous dynamical systems, so the autonomous case (which has neither been studied to our knowledge) will be a particular case also valid from our treatment.

In order to start our discussion for the theory of pullback attractors for nonautonomous dynamical systems, we need to modify the concept of semiflow, since the initial time is just as important as the final time.

Instead of a family of one time-dependent maps $S(t)$, we need to use a process (also called a two-parameter semigroup) on a metric space $(X, d)$. This is a family of continuous mappings $S(t, \tau): X \rightarrow X, t \geq \tau$, with the properties $S(t, t) x=x$, and $S\left(t_{2}, t_{1}\right) S\left(t_{1}, t_{0}\right)=S\left(t_{2}, t_{0}\right)$ for all $t_{0} \leq t_{1} \leq t_{2}$.

The main ingredients to obtain an attractor without clear compactness conditions are the following:

Let us denote $\mathcal{P}(X)$ the family of all nonempty subsets of $X$, and suppose $\mathcal{D}$ is a nonempty class of parameterized sets $\widehat{D}=\{D(t): t \in \mathbb{R}\} \subset \mathcal{P}(X)$.

Definition 2 The process $S(t, \tau)$ is said to be pullback $\mathcal{D}$-asymptotically compact if for any $t \in \mathbb{R}$, any $\widehat{D} \in \mathcal{D}$, any sequence $\tau_{n} \rightarrow-\infty$, and any sequence $x_{n} \in D\left(\tau_{n}\right)$, the sequence $\left\{S\left(t, \tau_{n}\right) x_{n}\right\}$ is relatively compact in $X$.

Let us denote by $\operatorname{dist}\left(C_{1}, C_{2}\right)$ the Hausdorff semidistance between $C_{1}$ and $C_{2}$, i.e.

$$
\operatorname{dist}\left(C_{1}, C_{2}\right)=\sup _{x \in C_{1}} \inf _{y \in C_{2}} d(x, y) \quad \text { for } C_{1}, C_{2} \subset X
$$

The property given in Definition 2 is enough to prove in a standard way the following result:

Proposition 3 Let us assume that the process $S(t, \tau)$ is pullback $\mathcal{D}$-asymptotically compact. For each $\widehat{D} \in \mathcal{D}$, we define the omega-limit of $\widehat{D}$ at time $t$ as the set given by

$$
\Lambda(t, \widehat{D})=\bigcap_{\tau \leq t}\left(\overline{\bigcup_{s \leq \tau} S(t, s, D(s))}\right) .
$$

This set is a non-empty compact subset of $X$, it attracts $\widehat{D}$ in the pullback sense, i.e.:

$$
\lim _{\tau \rightarrow-\infty} \operatorname{dist}(S(t, \tau, D(\tau)), \Lambda(t, \widehat{D}))=0,
$$

and it is invariant for $S$, i.e.:

$$
S(t, \tau, \Lambda(\tau, \widehat{D}))=\Lambda(t, \widehat{D}) \quad \forall t \geq \tau .
$$

Definition 4 It is said that $\widehat{B}=\{B(t): t \in \mathbb{R}\} \in \mathcal{D}$ is pullback $\mathcal{D}$-absorbing for the process $S$ if for any $t \in \mathbb{R}$ and any $\widehat{D} \in \mathcal{D}$, there exists a $\tau_{0}(t, \widehat{D}) \leq t$ 
such that

$$
S(t, \tau) D(\tau) \subset B(t) \quad \text { for all } \tau \leq \tau_{0}(t, \widehat{D})
$$

Joining the concepts given in Definitions 2 and 4 we have the following result (cf. [1]):

Theorem 5 Let us suppose that the process $S$ is pullback $\mathcal{D}$-asymptotically compact, and that $\widehat{B}=\{B(t): t \in \mathbb{R}\} \in \mathcal{D}$ is a family of pullback $\mathcal{D}$-absorbing sets for $S$. Then, the family $\mathcal{A}=\{\mathcal{A}(t): t \in \mathbb{R}\} \subset \mathcal{P}(X)$ defined by

$$
\mathcal{A}(t)=\Lambda(\widehat{B}, t), \quad t \in \mathbb{R},
$$

has the following properties:

(1) the set $\mathcal{A}(t)$ is compact for any $t \in \mathbb{R}$,

(2) $\mathcal{A}$ is pullback $\mathcal{D}$-attracting, i.e.

$$
\lim _{\tau \rightarrow-\infty} \operatorname{dist}(S(t, \tau) D(\tau), \mathcal{A}(t))=0 \quad \text { for all } \widehat{D} \in \mathcal{D}
$$

(3) $\mathcal{A}$ is invariant, i.e.

$$
S(t, \tau) \mathcal{A}(\tau)=\mathcal{A}(t) \quad \text { for all } \tau \leq t
$$

(4) and

$$
\mathcal{A}(t)=\overline{\bigcup_{\widehat{D} \in \mathcal{D}} \Lambda(\widehat{D}, t)} \quad \text { for } t \in \mathbb{R}
$$

The family $\mathcal{A}$, called the global pullback $\mathcal{D}$-attractor for the process $S$, is minimal in the sense that if $\widehat{C}=\{C(t): t \in \mathbb{R}\} \subset \mathcal{P}(X)$ is a family of closed sets such that

$$
\lim _{\tau \rightarrow-\infty} \operatorname{dist}(S(t, \tau) B(\tau), C(t))=0
$$

then $\mathcal{A}(t) \subset C(t)$.

Remark 6 (i) If we assume that $B(t)$ is closed for all $t \in \mathbb{R}$, and the family $\mathcal{D}$ is inclusion-closed (i.e. if $\widehat{D} \in \mathcal{D}$, and $\widehat{D}^{\prime}=\left\{D^{\prime}(t): t \in \mathbb{R}\right\} \subset \mathcal{P}(X)$ with $D^{\prime}(t) \subset D(t)$ for all $t$, then $\left.\widehat{D}^{\prime} \in \mathcal{D}\right)$, then the pullback $\mathcal{D}$-attractor $\mathcal{A}$ belongs to $\mathcal{D}$, and it is the unique family in $\mathcal{D}$ satisfying properties (1)-(3) above.

(ii) An important feature in the above result is that the attractor, as properly claimed in the statement, is compact and invariant.

Without the assumption of existence of a pullback $\mathcal{D}$-absorbing family for $S$, from Proposition 3 one is only able to show that $A(t)=\overline{\bigcup_{D \in \mathcal{D}} \Lambda(t, D)}$ is a 
closed set, that attracts all elements from $\mathcal{D}$, but it is only positively invariant, i.e. $A(t) \subset S(t, \tau, A(\tau))$. To prove the other inclusion, a compactness assumption on $A(\tau)$ would suffice.

This last assumption holds in the framework of the theorem, and also if one considers a subclass $\mathcal{E} \subset \mathcal{D}$ as universe, which will be used below to show the existence of different kind of attractors. (cf. Corollary 21).

\section{Construction of the process and main result}

In order to apply the theory of the previous section, we need to construct a process associated to problem (2).

We consider the Hilbert space $M_{H}^{2}=H \times L_{H}^{2}$ with the norm

$$
\left\|\left(u^{0}, \varphi\right)\right\|_{M_{H}^{2}}^{2}=\left|u^{0}\right|^{2}+\int_{-h}^{0}|\varphi(s)|^{2} \mathrm{~d} s .
$$

Taking into account Theorem 1, we can consider the family of mappings $S(t, \tau): M_{H}^{2} \rightarrow M_{H}^{2}$ given by

$$
S(t, \tau)\left(u^{0}, \varphi\right)=\left(u\left(t, \tau, u^{0}, \varphi\right), u_{t}\left(\cdot, \tau, u^{0}, \varphi\right)\right), \quad\left(u^{0}, \varphi\right) \in M_{H}^{2}, t \geq \tau .
$$

For technical reasons it will also be convenient to consider the Hilbert space $M_{V}^{2}=H \times L_{V}^{2}$ with the norm

$$
\left\|\left(u^{0}, \varphi\right)\right\|_{M_{V}^{2}}^{2}=\left|u^{0}\right|^{2}+\int_{-h}^{0}\|\varphi(s)\|^{2} \mathrm{~d} s .
$$

Remark 7 Observe that when the elapsed time $t-\tau$ is bigger than $h, S(t, \tau)$ maps $M_{H}^{2}$ into $M_{V}^{2}$.

To check we have a properly continuous process, we give firstly the following result:

Lemma 8 Let $\left(u^{0}, \varphi\right),\left(v^{0}, \psi\right) \in M_{H}^{2}$, be two couples of initial data for problem (2). Under the assumptions of Theorem 1 , denote $u(\cdot)=u\left(\cdot, \tau, u^{0}, \varphi\right)$ and $v(\cdot)=v\left(\cdot, \tau, v^{0}, \psi\right)$ the corresponding solutions to $(2)$. Then,

$$
|u(t)-v(t)|^{2} \leq\left(\left|u^{0}-v^{0}\right|^{2}+\|\varphi-\psi\|_{L_{H}^{2}}^{2}\right) \exp \left(\int_{\tau}^{t}\left(\frac{1}{2 \nu}\|u(s)\|^{2}+C_{g}^{2}+1\right) \mathrm{d} s\right), \forall t \geq \tau,
$$

and

$$
\nu \int_{\tau}^{t}\|u(s)-v(s)\|^{2} \mathrm{~d} s \leq\left(\left|u^{0}-v^{0}\right|^{2}+\|\varphi-\psi\|_{L_{H}^{2}}^{2}\right) \times
$$




$$
\times\left[1+\left(\int_{\tau}^{t}\left(\frac{1}{\nu}\|u(s)\|^{2}+C_{g}^{2}+1\right) \mathrm{d} s\right) \exp \left(\int_{\tau}^{t}\left(\frac{1}{\nu}\|u(s)\|^{2}+C_{g}^{2}+1\right) \mathrm{d} s\right)\right], \forall t \geq \tau .
$$

Proof. Writing the equation for the difference of $u$ and $v$ we have

$$
\frac{\mathrm{d}}{\mathrm{d} t}(u-v)+\nu A(u-v)+B(u)-B(v)=g\left(t, u_{t}\right)-g\left(t, v_{t}\right) .
$$

Let us denote $w=u-v$. Then, for any $t>\tau$

$\frac{1}{2} \frac{\mathrm{d}}{\mathrm{d} t}|w(t)|^{2}+\nu\|w(t)\|^{2}+\langle B(u(t))-B(v(t)), w(t)\rangle=\left(g\left(t, u_{t}\right)-g\left(t, v_{t}\right), w(t)\right)$.

Using that $(B(u)-B(v), w)=b(w, u, w)$, and the estimate (non-optimal but more clear) $|b(w, u, w)| \leq|w|\|u\|\|\mid w\|$, one has

$$
\begin{aligned}
\frac{\mathrm{d}}{\mathrm{d} t}|w(t)|^{2}+2 \nu\|w(t)\|^{2} \leq & 2|w(t)|\|u(t)\|\|w(t)\| \\
& +2\left|g\left(t, u_{t}\right)-g\left(t, v_{t}\right) \| w(t)\right| .
\end{aligned}
$$

Young inequality gives

$$
\begin{gathered}
2|w|\|u\|\|w\| \leq \frac{1}{2 \nu}|w|^{2}\|u\|^{2}+2 \nu\|w\|^{2} \text { and } \\
2\left|g\left(t, u_{t}\right)-g\left(t, v_{t}\right)\right||w(t)| \leq \frac{1}{C_{g}^{2}}\left|g\left(t, u_{t}\right)-g\left(t, v_{t}\right)\right|^{2}+C_{g}^{2}|w|^{2} .
\end{gathered}
$$

Putting this in (7) and integrating in $[\tau, t]$ we obtain

$$
|w(t)|^{2}-|w(\tau)|^{2} \leq \int_{\tau}^{t}\left(\frac{1}{2 \nu}|w(s)|^{2}\|u(s)\|^{2}+\frac{1}{C_{g}^{2}}\left|g\left(s, u_{s}\right)-g\left(s, v_{s}\right)\right|^{2}+C_{g}^{2}|w(s)|^{2}\right) \mathrm{d} s .
$$

Taking into account (IV) one has

$$
\begin{aligned}
\int_{\tau}^{t}\left|g\left(s, u_{s}\right)-g\left(s, v_{s}\right)\right|^{2} \mathrm{~d} s & \leq C_{g}^{2} \int_{\tau-h}^{t}|u(s)-v(s)|^{2} \mathrm{~d} s \\
& =C_{g}^{2}\|\varphi-\psi\|_{L_{H}^{2}}^{2}+C_{g}^{2} \int_{\tau}^{t}|w(s)|^{2} \mathrm{~d} s .
\end{aligned}
$$

Now we deduce

$$
|w(t)|^{2} \leq\left|u^{0}-v^{0}\right|^{2}+\|\varphi-\psi\|_{L_{H}^{2}}^{2}+\int_{\tau}^{t}\left(\frac{1}{2 \nu}\|u(s)\|^{2}+C_{g}^{2}+1\right)|w(s)|^{2} \mathrm{~d} s, \forall t \geq \tau .
$$

Applying Gronwall lemma we obtain (5). Now, using (5) in (7) we deduce (6).

We are now able to establish, as an immediate consequence, the following result. 
Theorem 9 Under the assumptions of Theorem 1, the family of maps $S(\cdot, \cdot)$ defined in (4) is a process in $M_{H}^{2}$. In fact, $S(t, \tau): M_{H}^{2} \rightarrow M_{H}^{2}$ is locally Lipschitz continuous, i.e. for any $t \geq \tau$ and any bounded set $B \subset M_{H}^{2}$, there exists a constant $L(B, t, \tau)>0$ such that for any couple $\left(u^{0}, \phi\right),\left(v^{0}, \psi\right) \in B$

$$
\left\|S(t, \tau)\left(u^{0}, \phi\right)-S(t, \tau)\left(v^{0}, \psi\right)\right\|_{M_{H}^{2}} \leq L(B, t, \tau)\left\|\left(u^{0}, \phi\right)-\left(v^{0}, \psi\right)\right\|_{M_{H}^{2}} .
$$

Remark 10 Although it will not be useful here, let us observe that from estimates in Lemma 8 we can also conclude that $S$ defines a continuous process on $C_{H}$.

We will also need the following continuity result for $S$ in a weak sense.

Proposition 11 Let us suppose that conditions from Theorem 1 hold. Then, for any sequence such that

$$
\left(u^{0, n}, \phi^{0, n}\right) \rightarrow\left(u^{0}, \phi^{0}\right) \text { weakly in } M_{V}^{2}
$$

and

$$
\frac{\mathrm{d} \phi^{0, n}}{\mathrm{~d} s} \rightarrow \frac{\mathrm{d} \phi^{0}}{\mathrm{~d} s} \text { weakly in } L_{V^{\prime}}^{2},
$$

the following convergences hold for any $t \geq \tau$ and any open bounded subset $\mathcal{O} \subset \Omega$ :

$$
\begin{aligned}
& u\left(t, \tau, u^{0, n}, \phi^{0, n}\right) \rightarrow u\left(t, \tau, u^{0}, \phi^{0}\right) \text { weakly in } H, \\
& u\left(\cdot, \tau, u^{0, n}, \phi^{0, n}\right) \rightarrow u\left(\cdot, \tau, u^{0}, \phi^{0}\right) \text { weakly in } L^{2}(\tau-h, t ; V), \\
& u_{t}\left(\cdot, \tau, u^{0, n}, \phi^{0, n}\right) \rightarrow u_{t}\left(\cdot, \tau, u^{0}, \phi^{0}\right) \text { weakly in } L_{V}^{2}, \\
& u\left(\cdot, \tau, u^{0, n}, \phi^{0, n}\right) \rightarrow u\left(\cdot, \tau, u^{0}, \phi^{0}\right) \text { strongly in } L^{2}\left(\tau-h, t ;\left(L^{2}(\mathcal{O})\right)^{2}\right) .
\end{aligned}
$$

Proof. Let us fix $t>\tau$ and a bounded open subset $\mathcal{O} \subset \Omega$. Denote for short $u^{n}(\cdot)=u\left(\cdot, \tau, u^{0, n}, \phi^{0, n}\right)$, and $u(\cdot)=u\left(\cdot, \tau, u^{0}, \phi^{0}\right)$ the corresponding solutions to problem (2). Observe that by Lemma 8 one has uniform bounds of $u^{n}$ and $u$ in $L^{\infty}(\tau, t ; H)$ and $L^{2}(\tau, t ; V)$. Observe that $u^{n}$ belongs to $C([\tau, t] ; H)$, so there exists $\xi \in H$ and $v \in L^{2}(\tau, t ; V)$ such that, for a subsequence $\left\{u^{n^{\prime}}\right\} \subset\left\{u^{n}\right\}$,

$$
u^{n^{\prime}}(t) \rightarrow \xi \text { weakly in } H
$$

and

$$
u^{n^{\prime}} \rightarrow v \text { weakly in } L^{2}(\tau, t ; V) .
$$

Observe also that analogously to $[14,16,8]$ we have that $\left.\phi^{0, n}\right|_{\mathcal{O}}$ is relatively compact in the strong topology of $L^{2}\left(-h, 0 ;\left(L^{2}(\mathcal{O})\right)^{2}\right)$.

Proceeding similarly to the existence theorem in [8] we see that $\xi=u(t)$ (in order to prove it, take a continuous differentiable function $\psi$ with $\psi(t) \neq 0$, and compare the obtained equalities). This proves (11). 
Property (12) comes from the fact that $u^{n^{\prime}} \rightarrow v$ weakly in $L^{2}(\tau, t ; V)$ and the uniqueness of solution corresponding to initial data $\left(u^{0}, \phi^{0}\right)$. Therefore, $v=u$ over $(\tau, t)$. Now, (13) is a trivial consequence of (12). Reasoning as in Lemma 2.4 in [8] we can also obtain that from $\left\{u^{n^{\prime}}\right\}$ we can extract a subsequence $\left\{u^{n^{\prime \prime}}\right\}$ satisfying (14). Finally, that (11)-(14) hold for the whole sequence can be seen by a contradiction argument taking into account the uniqueness of $u$.

\subsection{Existence of a bounded absorbing set}

In order to obtain a bounded absorbing set for the dynamical system $S$ in an adequate universe, we have the following result.

Proposition 12 Let us suppose that assumptions in Theorem 1 and the inequality $\nu \lambda_{1}>C_{g}$ hold. Assume also that there exists a value $m \in\left(0,2 \nu \lambda_{1}-2 C_{g}\right)$ such that for every $u \in L^{2}(\tau-h, t ; H)$,

$$
\int_{\tau}^{t} \mathrm{e}^{m s}\left|g\left(s, u_{s}\right)\right|^{2} \mathrm{~d} s \leq C_{g}^{2} \int_{\tau-h}^{t} \mathrm{e}^{m s}|u(s)|^{2} \mathrm{~d} s, \text { for any } t \geq \tau .
$$

Then, if we fix $\beta \in\left(0,2 \nu-2 C_{g} \lambda_{1}^{-1}-m \lambda_{1}^{-1}\right)$, and we denote

$$
\eta:=2 \nu-2 C_{g} \lambda_{1}^{-1}-m \lambda_{1}^{-1}-\beta>0,
$$

we have the inequality

$$
\begin{aligned}
& \left|u\left(t, \tau, u^{0}, \phi\right)\right|^{2}+\eta \mathrm{e}^{-m t} \int_{\tau}^{t} \mathrm{e}^{m s}\left\|u\left(s, \tau, u^{0}, \phi\right)\right\|^{2} \mathrm{~d} s \\
\leq & \mathrm{e}^{m(\tau-t)}\left(1 \vee 2 C_{g}\right)\left\|\left(u^{0}, \phi\right)\right\|_{M_{H}^{2}}^{2}+\beta^{-1} \mathrm{e}^{-m t} \int_{\tau}^{t} \mathrm{e}^{m s}\|f(s)\|_{*}^{2} \mathrm{~d} s
\end{aligned}
$$

for all $t \geq \tau$ and any $\left(u^{0}, \phi\right) \in M_{H}^{2}$.

Proof. Let us denote $u(t)=u\left(t, \tau, u^{0}, \phi\right)$. From the equation satisfied by $u(t)$ we have

$$
\begin{aligned}
\frac{\mathrm{d}}{\mathrm{d} t}\left(\mathrm{e}^{m t}|u(t)|^{2}\right)= & m \mathrm{e}^{m t}|u(t)|^{2}-2 \nu \mathrm{e}^{m t}\|u(t)\|^{2} \\
& +2 \mathrm{e}^{m t}\langle f(t), u(t)\rangle+2 \mathrm{e}^{m t}\left(g\left(t, u_{t}\right), u(t)\right) .
\end{aligned}
$$

By Young inequality, with constant $\beta$ to be fixed later on,

$$
2 \mathrm{e}^{m t}\langle f(t), u(t)\rangle \leq \beta^{-1} \mathrm{e}^{m t}\|f(t)\|_{*}^{2}+\mathrm{e}^{m t} \beta\|u(t)\|^{2},
$$


and then, if we integrate in $[\tau, t]$, we obtain

$$
\begin{aligned}
\mathrm{e}^{m t}|u(t)|^{2} \leq & \mathrm{e}^{m \tau}|u(\tau)|^{2}+\left(\frac{m}{\lambda_{1}}-2 \nu+\beta\right) \int_{\tau}^{t} \mathrm{e}^{m s}\|u(s)\|^{2} \mathrm{~d} s \\
& +\beta^{-1} \int_{\tau}^{t} \mathrm{e}^{m s}\|f(s)\|_{*}^{2} \mathrm{~d} s+2 \int_{\tau}^{t} \mathrm{e}^{m s}\left(g\left(s, u_{s}\right), u(s)\right) \mathrm{d} s .
\end{aligned}
$$

Observe that thanks to (15) the last term can be majorized by

$$
\begin{aligned}
2 \int_{\tau}^{t} \mathrm{e}^{m s}\left(g\left(s, u_{s}\right), u(s)\right) \mathrm{d} s & \leq 2\left(\int_{\tau}^{t} \mathrm{e}^{m s}\left|g\left(s, u_{s}\right)\right|^{2} \mathrm{~d} s\right)^{1 / 2}\left(\int_{\tau}^{t} \mathrm{e}^{m s}|u(s)|^{2} \mathrm{~d} s\right)^{1 / 2} \\
& \leq 2 C_{g} \int_{\tau-h}^{t} \mathrm{e}^{m s}|u(s)|^{2} \mathrm{~d} s \\
& \leq 2 C_{g} \mathrm{e}^{m \tau}\|\phi\|_{L_{H}^{2}}^{2}+2 C_{g} \int_{\tau}^{t} \mathrm{e}^{m s}|u(s)|^{2} \mathrm{~d} s
\end{aligned}
$$

Therefore, we conclude that

$$
\begin{aligned}
\mathrm{e}^{m t}|u(t)|^{2} \leq & \mathrm{e}^{m \tau}|u(\tau)|^{2}+2 C_{g} \mathrm{e}^{m \tau}\|\phi\|_{L_{H}^{2}}^{2} \\
& +\left(m \lambda_{1}^{-1}-2 \nu+\beta+2 C_{g} \lambda_{1}^{-1}\right) \int_{\tau}^{t} \mathrm{e}^{m s}\|u(s)\|^{2} \mathrm{~d} s \\
& +\beta^{-1} \int_{\tau}^{t} \mathrm{e}^{m s}\|f(s)\|_{*}^{2} \mathrm{~d} s,
\end{aligned}
$$

whence the result follows.

As a consequence of Proposition 12, we obtain the following estimate.

Corollary 13 Let us assume all the assumptions and notations in Proposition 12. Then, for any $r>0$ and any $\tau \leq t-r$, we have

$$
\begin{aligned}
\eta \int_{t-r}^{t}\left\|u\left(s, \tau, u^{0}, \phi\right)\right\|^{2} \mathrm{~d} s \leq & \mathrm{e}^{m(r+\tau-t)}\left(1 \vee 2 C_{g}\right)\left\|\left(u^{0}, \phi\right)\right\|_{M_{H}^{2}}^{2} \\
& +\beta^{-1} \mathrm{e}^{m(r-t)} \int_{\tau}^{t} \mathrm{e}^{m s}\|f(s)\|_{*}^{2} \mathrm{~d} s
\end{aligned}
$$

for any $\left(u^{0}, \phi\right) \in M_{H}^{2}$, where $\eta$ is given by (16)

Proof. Let us fix $r>0, \tau \leq t-r,\left(u^{0}, \phi\right) \in M_{H}^{2}$, and denote $u(\cdot)=$ $u\left(\cdot, \tau, u^{0}, \phi\right)$.

From (17) we obtain in particular that

$$
\eta \int_{\tau}^{t} \mathrm{e}^{m s}\|u(s)\|^{2} \mathrm{~d} s \leq \mathrm{e}^{m \tau}\left(1 \vee 2 C_{g}\right)\left\|\left(u^{0}, \phi\right)\right\|_{M_{H}^{2}}^{2}+\beta^{-1} \int_{\tau}^{t} \mathrm{e}^{m s}\|f(s)\|_{*}^{2} \mathrm{~d} s .
$$


But, as $\tau \leq t-r$,

$$
\begin{aligned}
\int_{\tau}^{t} \mathrm{e}^{m s}\|u(s)\|^{2} \mathrm{~d} s & \geq \int_{t-r}^{t} \mathrm{e}^{m s}\|u(s)\|^{2} \mathrm{~d} s \\
& \geq \mathrm{e}^{m(t-r)} \int_{t-r}^{t}\|u(s)\|^{2} \mathrm{~d} s
\end{aligned}
$$

and consequently we obtain (18).

From now on, we will assume all the assumptions in Proposition 12, and moreover that $f$ satisfies

$$
\int_{-\infty}^{t} \mathrm{e}^{m s}\|f(s)\|_{*}^{2} \mathrm{~d} s<+\infty \quad \text { for all } t \in \mathbb{R}
$$

We now define the universe $\mathcal{D}_{m}$ of tempered set we will use.

Definition 14 Let $\mathcal{R}_{m}$ be the set of all functions $r: \mathbb{R} \rightarrow(0,+\infty)$ such that

$$
\lim _{t \rightarrow-\infty} \mathrm{e}^{m t} r^{2}(t)=0 \text {. }
$$

We will denote by $\mathcal{D}_{m}$ the class of all families $\widehat{D}=\{D(t): t \in \mathbb{R}\} \subset \mathcal{P}\left(M_{H}^{2}\right)$ such that $D(t) \subset \bar{B}_{M_{H}^{2}}\left(0, r_{\widehat{\mathcal{D}}}(t)\right)$, for some $r_{\widehat{\mathcal{D}}} \in \mathcal{R}_{m}$, where $\bar{B}_{M_{H}^{2}}\left(0, r_{\widehat{\mathcal{D}}}(t)\right)$ denotes the closed ball in $M_{H}^{2}$ centered at zero with radius $r_{\widehat{\mathcal{D}}}(t)$.

Observe that $\mathcal{D}_{m}$ is inclusion-closed. Now, we can state the main result in this section, about the existence of an adequate $\mathcal{D}_{m}$-pullback absorbing set for the process $S(\cdot, \cdot)$ defined by $(4)$. Although it is immediate from Proposition 12 that the family $\widetilde{B}_{m}=\left\{B_{M_{H}^{2}}\left(0, \widetilde{R}_{m}(t)\right)\right\}_{t \in \mathbb{R}}$, with

$$
\widetilde{R}_{m}^{2}(t)=\beta^{-1}\left(1+h \mathrm{e}^{m h}\right) \mathrm{e}^{-m t} \int_{-\infty}^{t} \mathrm{e}^{m s}\|f(s)\|_{*}^{2} \mathrm{~d} s+1,
$$

verifies such condition, we will need, namely to apply Proposition 11, to obtain a different $\mathcal{D}_{m}$-pullback absorbing set.

Proposition 15 Under the assumptions and notation of Proposition 12, suppose that $f$ satisfies (20), and define

$$
r_{m}(t)=\beta^{-1} \mathrm{e}^{-m t} \int_{-\infty}^{t} \mathrm{e}^{m s}\|f(s)\|_{*}^{2} \mathrm{~d} s .
$$

Then, the family $\widehat{B}_{m}$ given by

$B_{m}(t)=\left\{\left(v^{0}, \psi\right) \in M_{V}^{2}:\left\|\left(v^{0}, \psi\right)\right\|_{M_{V}^{2}} \leq R_{m}(t), \quad\left\|\frac{\mathrm{d} \psi}{\mathrm{d} s}\right\|_{L_{V^{\prime}}^{2}} \leq \rho_{m}(t)\right\} \quad t \in \mathbb{R}$, 
with $R_{m}(t)>0$ and $\rho_{m}(t)>0$ defined respectively by

$$
\begin{aligned}
R_{m}^{2}(t)= & 1+\left(1+\eta^{-1} \mathrm{e}^{m h}\right) r_{m}(t), \\
\rho_{m}^{2}(t)= & 4 \int_{t-h}^{t}\|f(s)\|_{*}^{2} \mathrm{~d} s \\
& +4 \eta^{-1} \mathrm{e}^{2 m h} r_{m}(t)\left(\nu^{2}+C_{g}^{2} \lambda_{1}^{-2}+r_{m}(t)\right)+1,
\end{aligned}
$$

is pullback $\mathcal{D}_{m}$-absorbing for the process $S(\cdot, \cdot)$ defined by (4).

Proof. Observe that for any $t \in \mathbb{R}$,

$$
B_{m}(t) \subset\left\{\left(v^{0}, \psi\right) \in M_{H}^{2}:\left\|\left(v^{0}, \psi\right)\right\|_{M_{H}^{2}}^{2} \leq R_{m}^{2}(t)\right\},
$$

with

$$
\lim _{t \rightarrow-\infty} R_{m}^{2}(t) \mathrm{e}^{m t}=0
$$

and therefore $\widehat{B}_{m} \in \mathcal{D}_{m}$.

Let us fix $t \in \mathbb{R}$. The first part of the claim, that concerns the asymptotic estimate using $R_{m}(t)$, can be proved as follows.

From Corollary 13, taking $r=h$, we obtain that for any $\tau \leq t-h$, we have

$$
\begin{aligned}
\left\|u_{t}\left(\cdot, \tau, u^{0}, \phi\right)\right\|_{L_{V}^{2}}^{2} \leq & \eta^{-1} \mathrm{e}^{m(h+\tau-t)}\left(1 \vee 2 C_{g}\right)\left\|\left(u^{0}, \phi\right)\right\|_{M_{H}^{2}}^{2} \\
& +\eta^{-1} \beta^{-1} \mathrm{e}^{m(h-t)} \int_{-\infty}^{t} \mathrm{e}^{m s}\|f(s)\|_{*}^{2} \mathrm{~d} s
\end{aligned}
$$

for any $\left(u^{0}, \phi\right) \in M_{H}^{2}$.

From this inequality, (17) and the definition of $R_{m}(t)$, we obtain

$$
\left\|S(t, \tau)\left(u^{0}, \phi\right)\right\|_{M_{V}^{2}}^{2} \leq\left(1+\eta^{-1} \mathrm{e}^{m h}\right) \mathrm{e}^{m(\tau-t)}\left(1 \vee 2 C_{g}\right)\left\|\left(u^{0}, \phi\right)\right\|_{M_{H}^{2}}^{2}+R_{m}^{2}(t)-1
$$

for all $\tau \leq t-h$ and any $\left(u^{0}, \phi\right) \in M_{H}^{2}$.

Now, for the second part of the claim, that concerns the asymptotic estimate using $\rho_{m}(t)$, let us suppose that $\tau \leq t-2 h$ and denote $u(t)=u\left(t, \tau, u^{0}, \phi\right)$. From the equation satisfied by $u$, we immediately have

$$
\begin{aligned}
\left\|\frac{\mathrm{d} u(s)}{\mathrm{d} s}\right\|_{*} & \leq \nu\|u(s)\|+\|B(u(s))\|_{*}+\|f(s)\|_{*}+\left\|g\left(s, u_{s}\right)\right\|_{*} \\
& \leq \nu\|u(s)\|+|u(s)|\|u(s)\|+\|f(s)\|_{*}+\lambda_{1}^{-1 / 2}\left|g\left(s, u_{s}\right)\right|, \quad s \geq \tau .
\end{aligned}
$$


Therefore,

$$
\begin{aligned}
\int_{t-h}^{t}\left\|\frac{\mathrm{d} u(s)}{\mathrm{d} s}\right\|_{*}^{2} \mathrm{~d} s \leq \int_{t-h}^{t}( & 4 \nu^{2}\|u(s)\|^{2}+4|u(s)|^{2}\|u(s)\|^{2} \\
& \left.+4\|f(s)\|_{*}^{2}+4 \lambda_{1}^{-1}\left|g\left(s, u_{s}\right)\right|^{2}\right) \mathrm{d} s .
\end{aligned}
$$

Observe that by (II) and (IV),

$$
\int_{t-h}^{t}\left|g\left(s, u_{s}\right)\right|^{2} \mathrm{~d} s \leq C_{g}^{2} \lambda_{1}^{-1} \int_{t-2 h}^{t}\|u(s)\|^{2} \mathrm{~d} s,
$$

and thus, by (24) we have

$$
\begin{aligned}
\int_{t-h}^{t}\left\|\frac{\mathrm{d} u(s)}{\mathrm{d} s}\right\|_{*}^{2} \mathrm{~d} s \leq & 4 \int_{t-h}^{t}\|f(s)\|_{*}^{2} \mathrm{~d} s+4 \int_{t-h}^{t}|u(s)|^{2}\|u(s)\|^{2} \mathrm{~d} s \\
& +4\left(\nu^{2}+C_{g}^{2} \lambda_{1}^{-2}\right) \int_{t-2 h}^{t}\|u(s)\|^{2} \mathrm{~d} s
\end{aligned}
$$

Now observe that by (17), for all $s \in[t-h, t]$ one has

$$
\begin{aligned}
|u(s)|^{2} & \leq \mathrm{e}^{m(\tau-s)}\left(1 \vee 2 C_{g}\right)\left\|\left(u^{0}, \phi\right)\right\|_{M_{H}^{2}}^{2}+\beta^{-1} \mathrm{e}^{-m s} \int_{\tau}^{s} \mathrm{e}^{m r}\|f(r)\|_{*}^{2} \mathrm{~d} r \\
& \leq \mathrm{e}^{m h} \mathrm{e}^{m(\tau-t)}\left(1 \vee 2 C_{g}\right)\left\|\left(u^{0}, \phi\right)\right\|_{M_{H}^{2}}^{2}+\mathrm{e}^{m h} r_{m}(t) .
\end{aligned}
$$

Also, by Corollary 13 with $r=2 h$, we have

$$
\int_{t-2 h}^{t}\|u(s)\|^{2} \mathrm{~d} s \leq \eta^{-1} \mathrm{e}^{2 m h} \mathrm{e}^{m(\tau-t)}\left(1 \vee 2 C_{g}\right)\left\|\left(u^{0}, \phi\right)\right\|_{M_{H}^{2}}^{2}+\eta^{-1} \mathrm{e}^{2 m h} r_{m}(t) .
$$

Thus, if we denote

$$
C\left(t, \tau, u^{0}, \phi\right)=\mathrm{e}^{m h} \mathrm{e}^{m(\tau-t)}\left(1 \vee 2 C_{g}\right)\left\|\left(u^{0}, \phi\right)\right\|_{M_{H}^{2}}^{2}+\mathrm{e}^{m h} r_{m}(t),
$$

we deduce from (22), (25), (26) and (27) that

$$
\begin{array}{rl}
\left\|\frac{\mathrm{d}}{\mathrm{d} s} u_{t}\left(s, \tau, u^{0}, \phi\right)\right\|_{L_{V^{\prime}}^{2} \leq}^{2} & 4 \eta^{-1} C\left(t, \tau, u^{0}, \phi\right)\left[\mathrm{e}^{m h}\left(\nu^{2}+C_{g}^{2} \lambda_{1}^{-2}\right)+C\left(t, \tau, u^{0}, \phi\right)\right] \\
& +4 \int_{t-h}^{t}\|f(s)\|_{*}^{2} \mathrm{~d} s
\end{array}
$$

for all $\tau \leq t-2 h$ and any $\left(u^{0}, \phi\right) \in M_{H}^{2}$. 
Now, it is easy to see from $(23),(28)$ and the definition of $\mathcal{D}_{m}$, that the family $\widehat{B}_{m}$ given by $(21)$ is pullback $\mathcal{D}_{m}$-absorbing for the process $S(\cdot, \cdot)$.

\subsection{Asymptotic compactness}

In this section we prove, under an additional assumption on $g$, that the process $S$ is pullback $\mathcal{D}_{m}$-asymptotically compact. We will assume that $g$ also satisfies:

(VI) If $\left(\bar{u}^{0, n}, \bar{\phi}^{n}\right) \rightarrow\left(\bar{u}^{0}, \bar{\phi}\right)$ weakly in $M_{V}^{2}$ and $\frac{\mathrm{d} \bar{\phi}^{n}}{\mathrm{~d} s} \rightarrow \frac{\mathrm{d} \bar{\phi}}{\mathrm{d} s}$ weakly in $L_{V^{\prime}}^{2}$, then

$$
\begin{gathered}
\lim _{n \rightarrow+\infty} \int_{a}^{b}\left(g\left(s, u_{s}\left(\cdot, a, \bar{u}^{0, n}, \bar{\phi}^{n}\right)\right), u\left(s, a, \bar{u}^{0, n}, \bar{\phi}^{n}\right)\right) \mathrm{d} s \\
=\int_{a}^{b}\left(g\left(s, u_{s}\left(\cdot, a, \bar{u}^{0}, \bar{\phi}\right)\right), u\left(s, a, \bar{u}^{0}, \bar{\phi}\right)\right) \mathrm{d} s,
\end{gathered}
$$

for any $a<b$.

Remark 16 In our framework, condition (VI) is satisfied for instance if $g$ acts on a bounded set, that is, it contains as a multiplicative term the characteristic function $\chi_{\mathcal{O}}$, with $\mathcal{O}$ a bounded set of $\Omega$.

For example, when the function $g$ is defined by $g(t, \xi)(x)=\chi_{\mathcal{O}}(x) G(\xi(-\rho(t)(x)))$, for a suitable differentiable delay function $\rho$ and a globally Lipschitz continuous mapping $G: \mathbb{R}^{2} \rightarrow \mathbb{R}^{2}$, all the assumptions above hold (see [2] for more details and examples).

We will make use of the next simple auxiliary result:

Lemma 17 Suppose that $v^{n}$ converges to zero weakly in $L^{2}(a, b ; V)$ and strongly in $L^{2}\left(a, b ; V^{\prime}\right)$. Then, it also converges strongly to zero in $L^{2}(a, b ; H)$.

Proof. It is immediate, taking into account that

$$
\int_{a}^{b}\left|v^{n}(s)\right|^{2} \mathrm{~d} s=\int_{a}^{b}\left|\left\langle v^{n}(s), v^{n}(s)\right\rangle\right| \mathrm{d} s \leq\left\|v^{n}\right\|_{L^{2}\left(a, b ; V^{\prime}\right)}\left\|v^{n}\right\|_{L^{2}(a, b ; V)} .
$$

Proposition 18 Suppose the assumptions in Proposition 15 and that $g$ satisfies (VI). Then, the process $S$ defined by (4) is pullback $\mathcal{D}_{m}$-asymptotically compact.

Proof. Let $t \in \mathbb{R}, \widehat{D} \in \mathcal{D}_{m}$, a sequence $\tau_{n} \rightarrow-\infty$, and a sequence $\left(u^{0, n}, \phi^{n}\right) \in$ $D\left(\tau_{n}\right)$, be fixed. We must prove that the sequence

$$
S\left(t, \tau_{n}\right)\left(u^{0, n}, \phi^{n}\right)=\left(u\left(t, \tau_{n},\left(u^{0, n}, \phi^{n}\right)\right), u_{t}\left(\cdot, \tau_{n},\left(u^{0, n}, \phi^{n}\right)\right)\right)
$$


is relatively compact in $M_{H}^{2}$. We proceed in two steps.

Step 1: We check similarly to [1] the asymptotic compactness in the first component of $S$.

By Proposition 15 we know that $\widehat{B}_{m}$ defined by (21) is pullback $\mathcal{D}_{m}$-absorbing for $S(\cdot, \cdot): M_{H}^{2} \rightarrow M_{H}^{2}$. So, for each integer $k \geq 0$ there exists $\tau_{\widehat{D}}(k) \leq t-k$ such that

$$
S(t-k, \tau) D(\tau) \subset B_{m}(t-k), \forall \tau \leq \tau_{\widehat{D}}(k) .
$$

From (29) and a diagonal argument, we can extract a subsequence $\left\{\left(u^{0, n^{\prime}}, \phi^{n^{\prime}}\right)\right\} \subset$ $\left\{\left(u^{0, n}, \phi^{n}\right)\right\}$ such that for each integer $k$

$$
\begin{gathered}
S\left(t-k, \tau_{n^{\prime}}\right)\left(u^{0, n^{\prime}}, \phi^{n^{\prime}}\right) \rightarrow\left(w^{k}, \psi^{k}\right) \quad \text { weakly in } M_{V}^{2}, \\
\frac{\mathrm{d}}{\mathrm{d} s} u_{t-k}\left(s, \tau_{n^{\prime}}\right)\left(u^{0, n^{\prime}}, \phi^{n^{\prime}}\right) \rightarrow \frac{\mathrm{d}}{\mathrm{d} s} \psi^{k} \quad \text { weakly in } M_{V^{\prime}}^{2},
\end{gathered}
$$

with each $\left(w^{k}, \psi^{k}\right) \in B_{m}(t-k)$.

Now, we may apply Proposition 11 on each fixed interval $[t-k, t]$ to deduce that

$$
\begin{aligned}
\left(w^{0}, \phi^{0}\right) & =M_{V}^{2}-\text { weak } \lim _{n^{\prime} \rightarrow+\infty} S\left(t, \tau_{n^{\prime}}\right)\left(u^{0, n^{\prime}}, \phi^{n^{\prime}}\right) \\
& =M_{V}^{2}-\text { weak } \lim _{n^{\prime} \rightarrow+\infty} S(t, t-k) S\left(t-k, \tau_{n^{\prime}}\right)\left(u^{0, n^{\prime}}, \phi^{n^{\prime}}\right) \\
& =S(t, t-k)\left[M_{V}^{2}-\text { weak } \lim _{n^{\prime} \rightarrow+\infty} S\left(t-k, \tau_{n^{\prime}}\right)\left(u^{0, n^{\prime}}, \phi^{n^{\prime}}\right)\right] \\
& =S(t, t-k)\left(w^{k}, \psi^{k}\right) .
\end{aligned}
$$

From (30) with $k=0$ we obtain in particular that

$$
\left|w^{0}\right| \leq \liminf _{n^{\prime} \rightarrow+\infty}\left|u\left(t, \tau_{n^{\prime}}, u^{0, n^{\prime}}, \phi^{n^{\prime}}\right)\right|
$$

We will prove now that $\lim \sup _{n^{\prime} \rightarrow+\infty}\left|u\left(t, \tau_{n^{\prime}}, u^{0, n^{\prime}}, \phi^{n^{\prime}}\right)\right| \leq\left|w^{0}\right|$, and therefore conclude (with the weak limit) that the limit is in the strong topology of $H$. After that, we will use it to prove that (30) holds (for another subsequence) also in the strong sense and not only in a weak sense.

Following $[14,1]$ we will denote here

$$
[u]^{2}=\nu\|u\|^{2}-\frac{m}{2}|u|^{2},
$$

which, taking into account that $m<2 \nu \lambda_{1}$, is a Hilbert norm in $V$ equivalent to $\|u\|$. 
From (2) we deduce that for an arbitrary solution $u\left(\cdot, \tau, \bar{u}^{0}, \bar{\phi}\right)$ one has

$$
\begin{aligned}
\left|u\left(t, \tau, \bar{u}^{0}, \bar{\phi}\right)\right|^{2}= & \mathrm{e}^{m(\tau-t)}\left|\bar{u}^{0}\right|^{2}+2 \int_{\tau}^{t} \mathrm{e}^{m(s-t)}\left\{\left\langle f(s), u\left(s, \tau, \bar{u}^{0}, \bar{\phi}\right)\right\rangle\right. \\
& \left.+\left(g\left(s, u_{s}\left(\cdot, \tau, \bar{u}^{0}, \bar{\phi}\right)\right), u\left(s, \tau, \bar{u}^{0}, \bar{\phi}\right)\right)-\left[u\left(s, \tau, \bar{u}^{0}, \bar{\phi}\right)\right]^{2}\right\} \mathrm{d} s .
\end{aligned}
$$

Consider any fixed value $k$ and so for all $\tau_{n^{\prime}} \leq t-k$, applying (34), one has

$$
\begin{aligned}
\left|u\left(t, \tau_{n^{\prime}}, u^{0, n^{\prime}}, \phi^{n^{\prime}}\right)\right|^{2}= & \left|u\left(t, t-k, S\left(t-k, \tau_{n^{\prime}}\right)\left(u^{0, n^{\prime}}, \phi^{n^{\prime}}\right)\right)\right|^{2} \\
= & \mathrm{e}^{-m k}\left|u\left(t-k, \tau_{n^{\prime}}, u^{0, n^{\prime}}, \phi^{n^{\prime}}\right)\right|^{2} \\
& +2 \int_{t-k}^{t} \mathrm{e}^{m(s-t)}\left\langle f(s), u\left(s, t-k, S\left(t-k, \tau_{n^{\prime}}\right)\left(u^{0, n^{\prime}}, \phi^{n^{\prime}}\right)\right)\right\rangle \mathrm{d} s \\
& +2 \int_{t-k}^{t} \mathrm{e}^{m(s-t)}\left(g\left(s, u_{s}\left(\cdot, t-k, S\left(t-k, \tau_{n^{\prime}}\right)\left(u^{0, n^{\prime}}, \phi^{n^{\prime}}\right)\right)\right),\right. \\
& \left.u\left(s, t-k, S\left(t-k, \tau_{n^{\prime}}\right)\left(u^{0, n^{\prime}}, \phi^{n^{\prime}}\right)\right)\right) \mathrm{d} s \\
& -2 \int_{t-k}^{t} \mathrm{e}^{m(s-t)}\left[u\left(s, t-k, S\left(t-k, \tau_{n^{\prime}}\right)\left(u^{0, n^{\prime}}, \phi^{n^{\prime}}\right)\right)\right]^{2} \mathrm{~d} s .
\end{aligned}
$$

Since $S\left(t-k, \tau_{n^{\prime}}\right)\left(u^{0, n^{\prime}}, \phi^{n^{\prime}}\right) \in B_{m}(t-k)$ for all $\tau_{n^{\prime}} \leq \tau_{\tilde{D}}(k)$ for all $k \geq 0$, we have that

$$
\limsup _{n^{\prime} \rightarrow+\infty}\left\|S\left(t-k, \tau_{n^{\prime}}\right)\left(u^{0, n^{\prime}}, \phi^{n^{\prime}}\right)\right\|_{M_{V}^{2}}^{2} \leq R_{m}^{2}(t-k), \forall k \geq 0 .
$$

On other hand, by (30), (31) and Proposition 11 we deduce that

$u\left(\cdot, t-k, S\left(t-k, \tau_{n^{\prime}}\right)\left(u^{0, n^{\prime}}, \phi^{n^{\prime}}\right)\right) \rightarrow u\left(\cdot, t-k, w^{k}, \psi^{k}\right) \quad$ weakly in $L^{2}(t-k, t ; V)$.

As $\mathrm{e}^{m(\cdot-t)} f(\cdot) \in L^{2}\left(t-k, t ; V^{\prime}\right)$, we deduce that

$$
\begin{gathered}
\lim _{n^{\prime} \rightarrow+\infty} \int_{t-k}^{t} \mathrm{e}^{m(s-t)}\left\langle f(s), u\left(s, t-k, S\left(t-k, \tau_{n^{\prime}}\right)\left(u^{0, n^{\prime}}, \phi^{n^{\prime}}\right)\right)\right\rangle \mathrm{d} s \\
=\int_{t-k}^{t} \mathrm{e}^{m(s-t)}\left\langle f(s), u\left(s, t-k, w^{k}, \psi^{k}\right)\right\rangle \mathrm{d} s .
\end{gathered}
$$

As $\left(\int_{t-k}^{t} \mathrm{e}^{m(s-t)}[v(s)]^{2} \mathrm{~d} s\right)$ defines an equivalent norm in $L^{2}(t-k, t ; V)$, we deduce from (37) that

$$
\begin{aligned}
& \int_{t-k}^{t} \mathrm{e}^{m(s-t)}\left[u\left(s, t-k, w^{k}, \psi^{k}\right)\right]^{2} \mathrm{~d} s \\
\leq & \liminf _{n^{\prime} \rightarrow+\infty} \int_{t-k}^{t} \mathrm{e}^{m(s-t)}\left[u\left(s, t-k, S\left(t-k, \tau_{n^{\prime}}\right)\left(u^{0, n^{\prime}}, \phi^{n^{\prime}}\right)\right)\right]^{2} \mathrm{~d} s .
\end{aligned}
$$


Finally, taking into account condition (VI) we obtain that

$$
\begin{gathered}
\lim _{n^{\prime} \rightarrow+\infty} \int_{t-k}^{t} \mathrm{e}^{m(s-t)}\left(g\left(s, u_{s}\left(\cdot, t-k, S\left(t-k, \tau_{n^{\prime}}\right)\left(u^{0, n^{\prime}}, \phi^{n^{\prime}}\right)\right)\right),\right. \\
\left.u\left(s, t-k, S\left(t-k, \tau_{n^{\prime}}\right)\left(u^{0, n^{\prime}}, \phi^{n^{\prime}}\right)\right)\right) \mathrm{d} s \\
=\int_{t-k}^{t} \mathrm{e}^{m(s-t)}\left(g\left(s, u_{s}\left(\cdot, t-k, w^{k}, \psi^{k}\right)\right), u\left(s, t-k, w^{k}, \psi^{k}\right)\right) \mathrm{d} s .
\end{gathered}
$$

This equality, jointly with (35), (36), (38) and (39), give us that

$$
\begin{aligned}
& \limsup _{n^{\prime} \rightarrow+\infty}\left|u\left(t, \tau_{n^{\prime}}, u^{0, n^{\prime}}, \phi^{n^{\prime}}\right)\right|^{2} \\
\leq & \mathrm{e}^{-m k} R_{m}^{2}(t-k)+2 \int_{t-k}^{t} \mathrm{e}^{m(s-t)}\left\langle f(s), u\left(s, t-k, w^{k}, \psi^{k}\right)\right\rangle \mathrm{d} s \\
& +2 \int_{t-k}^{t} \mathrm{e}^{m(s-t)}\left(g\left(s, u_{s}\left(\cdot, t-k, w^{k}, \psi^{k}\right)\right), u\left(s, t-k, w^{k}, \psi^{k}\right)\right) \mathrm{d} s \\
& -2 \int_{t-k}^{t} \mathrm{e}^{m(s-t)}\left[u\left(s, t-k, w^{k}, \psi^{k}\right)\right]^{2} \mathrm{~d} s .
\end{aligned}
$$

Now, if we write equality (34) for $u\left(t, t-k, w^{k}, \psi^{k}\right)=w^{0}$ (remind (32)) we obtain

$$
\begin{aligned}
&\left|w^{0}\right|^{2}=\left|w^{k}\right|^{2} \mathrm{e}^{-m k} \\
&+2 \int_{t-k}^{t} \mathrm{e}^{m(s-t)}\left\{\left\langle f(s), u\left(s, t-k, w^{k}, \psi^{k}\right)\right\rangle\right. \\
& \quad+\left(g\left(s, u_{s}\left(\cdot, t-k, w^{k}, \psi^{k}\right)\right), u\left(s, t-k, w^{k}, \psi^{k}\right)\right) \\
&\left.\quad-\left[u\left(s, t-k, w^{k}, \psi^{k}\right)\right]^{2}\right\} \mathrm{d} s .
\end{aligned}
$$

Comparing (40) and (41) we deduce

$$
\limsup _{n^{\prime} \rightarrow+\infty}\left|u\left(t, \tau_{n^{\prime}}, u^{0, n^{\prime}}, \phi^{n^{\prime}}\right)\right|^{2} \leq \mathrm{e}^{-m k} R_{m}^{2}(t-k)-\mathrm{e}^{-m k}\left|w^{k}\right|^{2}+\left|w^{0}\right|^{2},
$$

and then, taking into account that

$$
\lim _{k \rightarrow+\infty} \mathrm{e}^{-m k} R_{m}^{2}(t-k)=0
$$

we obtain

$$
\limsup _{n^{\prime} \rightarrow+\infty}\left|u\left(t, \tau_{n^{\prime}}, u^{0, n^{\prime}}, \phi^{n^{\prime}}\right)\right|^{2} \leq\left|w^{0}\right|^{2} .
$$

From this inequality, jointly with (33) and the weak convergence of $u\left(t, \tau_{n^{\prime}}, u^{0, n^{\prime}}, \phi^{n^{\prime}}\right)$ to $w^{0}$ we deduce that

$$
u\left(t, \tau_{n^{\prime}}, u^{0, n^{\prime}}, \phi^{n^{\prime}}\right) \rightarrow w^{0} \quad \text { strongly in } H .
$$


Step 2: Now, we prove the asymptotic compactness in the second component of $\overline{S . M o r e}$ exactly, having in mind (30), we will prove that $\left\{u_{t}\left(\cdot, \tau_{n^{\prime}}, u^{0, n^{\prime}}, \phi^{n^{\prime}}\right)\right\}_{n^{\prime}}$ has a subsequence that converges in $L_{H}^{2}$ to $\psi^{0}$.

Let us denote $\left\{\theta_{j}: j \geq 0\right\}$ the sequence of all rational numbers from the interval $[-h, 0]$. Observe that reasoning as in Step 1, through a diagonal argument, we can obtain a subsequence, that we will continue denoting $\left(u^{0, n^{\prime}}, \phi^{n^{\prime}}\right)$, such that $\tau_{n^{\prime}} \leq t-2 h$ and for all $j$ there exists $\bar{w}^{j} \in H$ such that

$$
\lim _{n^{\prime} \rightarrow+\infty} u\left(t+\theta_{j}, \tau_{n^{\prime}}, u^{0, n^{\prime}}, \phi^{n^{\prime}}\right)=\bar{w}^{j} \text { in } H .
$$

We simply check now an equicontinuity property for the solutions $\left\{u\left(\cdot, \tau_{n^{\prime}}, u^{0, n^{\prime}}, \phi^{n^{\prime}}\right)\right\}$ in the interval $[t-h, t]$.

Denote $u^{n^{\prime}}(\cdot)=u\left(\cdot, \tau_{n^{\prime}}, u^{0, n^{\prime}}, \phi^{n^{\prime}}\right)$. Then, for any pair $t_{1}, t_{2} \in[t-h, t]$ with $t_{1}<t_{2}$ we have the equality in $V^{\prime}$ :

$$
u^{n^{\prime}}\left(t_{2}\right)-u^{n^{\prime}}\left(t_{1}\right)=\int_{t_{1}}^{t_{2}}\left(-\nu A u^{n^{\prime}}(s)-B\left(u^{n^{\prime}}(s)\right)+f(s)+g\left(s, u_{s}^{n^{\prime}}\right)\right) \mathrm{d} s .
$$

Therefore,

$$
\begin{aligned}
& \left\|u^{n^{\prime}}\left(t_{2}\right)-u^{n^{\prime}}\left(t_{1}\right)\right\|_{*} \\
\leq & \int_{t_{1}}^{t_{2}}\left(\nu\left\|u^{n^{\prime}}(s)\right\|+\left|u^{n^{\prime}}(s)\right|\left\|u^{n^{\prime}}(s)\right\|+\|f(s)\|_{*}+\left\|g\left(s, u_{s}^{n^{\prime}}\right)\right\|_{*}\right) \mathrm{d} s .
\end{aligned}
$$

Now it is easy to deduce from (II) and (IV) that

$$
\begin{aligned}
\int_{t_{1}}^{t_{2}}\left\|g\left(s, u_{s}^{n^{\prime}}\right)\right\|_{*} \mathrm{~d} s & \leq \lambda_{1}^{-1 / 2} \int_{t_{1}}^{t_{2}}\left|g\left(s, u_{s}^{n^{\prime}}\right)\right| \mathrm{d} s \\
& \leq C_{g} \lambda_{1}^{-1 / 2}\left(t_{2}-t_{1}\right)^{1 / 2}\left(\int_{t_{1}-h}^{t_{2}}\left|u^{n^{\prime}}(s)\right|^{2} \mathrm{~d} s\right)^{1 / 2} \\
& \leq C_{g} \lambda_{1}^{-1}\left(t_{2}-t_{1}\right)^{1 / 2}\left(\int_{t-2 h}^{t}\left\|u^{n^{\prime}}(s)\right\|^{2} \mathrm{~d} s\right)^{1 / 2} .
\end{aligned}
$$

Similarly, one has

and

$$
\begin{aligned}
& \int_{t_{1}}^{t_{2}}\|f(s)\|_{*} \mathrm{~d} s \leq\left(t_{2}-t_{1}\right)^{1 / 2}\left(\int_{t-h}^{t}\|f(s)\|_{*}^{2} \mathrm{~d} s\right)^{1 / 2}, \\
& \int_{t_{1}}^{t_{2}}\left\|u^{n^{\prime}}(s)\right\| \mathrm{d} s \leq\left(t_{2}-t_{1}\right)^{1 / 2}\left(\int_{t_{1}}^{t_{2}}\left\|u^{n^{\prime}}(s)\right\|^{2} \mathrm{~d} s\right)^{1 / 2}
\end{aligned}
$$

$$
\int_{t_{1}}^{t_{2}}\left|u^{n^{\prime}}(s)\right|\left\|u^{n^{\prime}}(s)\right\| \mathrm{d} s \leq\left(t_{2}-t_{1}\right)^{1 / 2} \sup _{[t-h, t]}\left|u^{n^{\prime}}(s)\right|\left(\int_{t_{1}}^{t_{2}}\left\|u^{n^{\prime}}(s)\right\|^{2} \mathrm{~d} s\right)^{1 / 2} .
$$


From these inequalities and (43) we deduce

$$
\begin{aligned}
\left\|u^{n^{\prime}}\left(t_{2}\right)-u^{n^{\prime}}\left(t_{1}\right)\right\|_{*} \leq & \left(t_{2}-t_{1}\right)^{1 / 2}\left[\left(\int_{t-h}^{t}\|f(s)\|_{*}^{2} \mathrm{~d} s\right)^{1 / 2}\right. \\
& \left.+\left(\nu+\sup _{[t-h, t]}\left|u^{n^{\prime}}(s)\right|+C_{g} \lambda_{1}^{-1}\right)\left(\int_{t-2 h}^{t}\left\|u^{n^{\prime}}(s)\right\|^{2} \mathrm{~d} s\right)^{1 / 2}\right],
\end{aligned}
$$

for all $t_{1}, t_{2} \in[t-h, t]$ with $t_{1}<t_{2}$.

Now, observe that from (17) we obtain in particular

$$
\begin{aligned}
\left|u^{n^{\prime}}(r)\right|^{2} \leq & \mathrm{e}^{m\left(\tau_{n^{\prime}}-r\right)}\left(1 \vee 2 C_{g}\right)\left\|\left(u^{0, n^{\prime}}, \phi^{n^{\prime}}\right)\right\|_{M_{H}^{2}}^{2} \\
& +\beta^{-1} \mathrm{e}^{-m r} \int_{-\infty}^{r} \mathrm{e}^{m s}\|f(s)\|_{*}^{2} \mathrm{~d} s \\
\leq & \mathrm{e}^{m\left(h+\tau_{n^{\prime}}-t\right)}\left(1 \vee 2 C_{g}\right)\left\|\left(u^{0, n^{\prime}}, \phi^{n^{\prime}}\right)\right\|_{M_{H}^{2}}^{2} \\
& +\beta^{-1} \mathrm{e}^{m(h-t)} \int_{-\infty}^{t} \mathrm{e}^{m s}\|f(s)\|_{*}^{2} \mathrm{~d} s,
\end{aligned}
$$

for all $r \in[t-h, t]$.

Analogously, we obtain from Corollary 13

$$
\begin{aligned}
\eta \int_{t-2 h}^{t}\left\|u^{n^{\prime}}(s)\right\|^{2} \mathrm{~d} s \leq & \mathrm{e}^{m\left(2 h+\tau_{n^{\prime}}-t\right)}\left(1 \vee 2 C_{g}\right)\left\|\left(u^{0, n^{\prime}}, \phi^{n^{\prime}}\right)\right\|_{M_{H}^{2}}^{2} \\
& +\beta^{-1} \mathrm{e}^{m(2 h-t)} \int_{-\infty}^{t} \mathrm{e}^{m s}\|f(s)\|_{*}^{2} \mathrm{~d} s .
\end{aligned}
$$

Taking into account that the sequence $\mathrm{e}^{m \tau_{n^{\prime}}}\left\|\left(u^{0, n^{\prime}}, \phi^{n^{\prime}}\right)\right\|_{M_{H}^{2}}^{2}$ is in particular bounded, we deduce from (44), (45) and (46), the equicontinuity in $C$ ([t$\left.h, t] ; V^{\prime}\right)$ of the sequence $\left\{u^{n^{\prime}}\right\}$.

Similarly to the Ascoli-Arzelà Theorem we can obtain now pointwise convergence: in order to show that for any $r \in[t-h, t] \backslash \mathbb{Q}$ the sequence $\left\{u^{n^{\prime}}(r)\right\}$ is a Cauchy sequence, observe that

$$
\begin{aligned}
\left\|u^{n^{\prime}}(r)-u^{m^{\prime}}(r)\right\|_{*} \leq & \left\|u^{n^{\prime}}(r)-u^{n^{\prime}}\left(t+\theta_{j}\right)\right\|_{*}+\left\|u^{n^{\prime}}\left(t+\theta_{j}\right)-u^{m^{\prime}}\left(t+\theta_{j}\right)\right\|_{*} \\
& +\left\|u^{m^{\prime}}\left(t+\theta_{j}\right)-u^{m^{\prime}}(r)\right\|_{*} .
\end{aligned}
$$

The first and third terms in the right hand side can be as small as wished by the equicontinuity property provided $\theta_{j}$ is suitably choosen. The second term provides a Cauchy sequence, for this fixed $t+\theta_{j}$, thanks to (42). 
Thus, we have obtained that for all $r \in[t-h, t]$ there exists

$$
V^{\prime}-\lim _{n^{\prime} \rightarrow+\infty} u^{n^{\prime}}(r)=: v(r) .
$$

Then, taking into account that by (45) and the continuous injection of $H$ into $V^{\prime}$, the sequence $\left\{u^{n^{\prime}}\right\}$ is uniformly bounded in $C\left([t-h, t] ; V^{\prime}\right)$, we can ensure by the Lebesgue Theorem that $u^{n^{\prime}}$ converges to $v$ stronlgy in $L^{2}\left(t-h, t ; V^{\prime}\right)$, i.e.,

$$
u_{t}\left(\cdot, \tau_{n^{\prime}}, u^{n^{\prime}}, \phi^{n^{\prime}}\right) \rightarrow v(t+\cdot) \quad \text { strongly in } L_{V^{\prime}}^{2} .
$$

Recalling the weak convergence (30), we obtain

$$
u_{t}\left(\cdot, \tau_{n^{\prime}}, u^{0, n^{\prime}}, \phi^{n^{\prime}}\right) \rightarrow \phi^{0} \quad \text { strongly in } L_{V^{\prime}}^{2} .
$$

The proof finishes taking into account (30), (48), and Lemma 17.

Remark 19 Indeed, the convergence (48) in the above proof holds in $C\left([-h, 0] ; V^{\prime}\right)$ (to see this, observe that $v$ is continuous, this is not difficult to check; and so the equality $v(t+\cdot)=\phi^{0}$ does hold pointwise, and not only a.e.).

Finally, we summarize the conclusions of the above sections and Theorem 5 in our main result: the existence of tempered pullback attractor in the universe $\mathcal{D}_{m}$ defined by (14).

Theorem 20 Let $f \in L_{l o c}^{2}\left(\mathbb{R} ; V^{\prime}\right)$, and $g: \mathbb{R} \times C_{H} \rightarrow\left(L^{2}(\Omega)\right)^{2}$ satisfying hypotheses (I)-(VI). Assume that $\nu \lambda_{1}>C_{g}$, and there exists $m \in\left(0,2 \nu \lambda_{1}-2 C_{g}\right)$ such that (15) and (20) are satisfied.

Then, there exists a unique global pullback $\mathcal{D}_{m}$-attractor for the process $S(\cdot, \cdot)$ belonging to $\mathcal{D}_{m}$.

Taking into account Remark 6, we can establish the following interesting byproduct:

Corollary 21 Under the assumptions of Theorem 20, and denoting $\mathcal{E}=\{D$ : $D$ bounded in $\left.\mathbb{R}^{2}\right\}$, there also exists a global pullback attractor for problem (2), in the sense given in Theorem 5 . Namely, $\mathcal{A}_{\mathcal{E}}(t)=\overline{\bigcup_{D \in \mathcal{E}} \Lambda(D, t)}$ is compact for any $t \in \mathbb{R}$, invariant, $S(t, \tau) \mathcal{A}_{\mathcal{E}}(\tau)=\mathcal{A}_{\mathcal{E}}(t)$ for all $\tau \leq t$, and attracts bounded sets in the pullback sense:

$$
\lim _{\tau \rightarrow-\infty} \operatorname{dist}\left(S(t, \tau) D, \mathcal{A}_{\mathcal{E}}(t)\right)=0 \quad \text { for all } D \in \mathcal{E}
$$

Remark 22 The above results can be immediately particularized to the autonomous case (i.e. without explicit dependence on time of $f$ and $g$ ) since then global and pullback attractors are equivalent. Actually, (20) holds trivially when $f$ is independent of time. 


\section{Acknowledgments}

The authors wish to thank the referee for his/her helpful and detailed suggestions. This work was partially supported by Ministerio de Educación y Ciencia (Spain) MTM2005-01412.

\section{References}

[1] T. Caraballo, G. Łukaszewicz, J. Real, Pullback attractors for asymptotically compact non-autonomous dynamical systems, Nonlinear Anal. 64 (2006), no. $3,484-498$.

[2] T. Caraballo, J. Real, Navier-Stokes equations with delays, R. Soc. Lond. Proc. Ser. A Math. Phys. Eng. Sci. 457 (2001), no. 2014, 2441-2453.

[3] T. Caraballo, J. Real, Asymptotic behaviour of two-dimensional Navier-Stokes equations with delays, R. Soc. Lond. Proc. 459 (2003), no. 2040, 3181-3194.

[4] T. Caraballo, J. Real, Attractors for 2D-Navier-Stokes models with delays, $J$. Differential Equations 205 (2004), no. 2, 271-297.

[5] V. Chepyzhov, M. Vishik, Attractors for equations of matehmatical physics, in: American Mathematical Society Colloquium Publications, Vol. 49, AMS, Providece, RI, 2002.

[6] P. Constantin, C. Foias, Navier Stokes Equations, The University of Chicago Press, Chicago, 1988.

[7] H. Crauel, A. Debussche, F. Flandoli, Random attractors, J. Dynam. Differential Equations 9 (1997), no.2, 307-341.

[8] M. J. Garrido-Atienza, P. Marín-Rubio, Navier-Stokes equations with delays on unbounded domains, Nonlinear Anal. 64 (2006), no 5, 1100-1118.

[9] J. K. Hale, Asymptotic Behavior of Dissipative Systems, Matehmatical Srveys and Monographs, AMS, Providence, RI, 1988.

[10] P. E. Kloeden, B. Schmalfuß, Nonautonomous systems, cocycle attractors and variable time-step discretization. Dynamical numerical analysis (Atlanta, GA, 1995). Numer. Algorithms 14 (1997), 141-152.

[11] O. Ladyzhenskaya, Attractors for Semigroups and Evolution Equations, Cambridge Univesity Press, Cambridge, 1991.

[12] O. Ladyzhenskaya, First boundary value problem for the Navier-Stokes equations in domains with nonsmooth boundaries. C. R. Acad. Sci. Paris Sér. I Math. 314 (1992), 253-258.

[13] I. Moise, R. Rosa, X. Wang, Attractors for noncompact nonautonomous systems via energy equations, Discrete Contin. Dyn. Syst. 10 (2004), no. 1-2,473-496. 
[14] R. Rosa, The global attractor for the 2D Navier-Stokes flow on some unbounded domains, Nonlinear Anal. 32 (1998), no 1, 71-85.

[15] B. Schmalfuß, Backward cocycles and attractors of stochastic differential equations, on International Seminar on Applied Mathematics-Nonlinear Dynamics: Attractor Approximation and Global Behaviour, Eds.:V. Reitmann and T. Redrich and N. J. Kosch, Dresden, Technische Universität, 1992, 185192.

[16] J. Simon, 2003 Équations de Navier-Stokes, Cours de DEA 2002-2003, Université Blaise Pascal. http://wwwlma.univ-bpclermont.fr/ simon/

[17] R. Temam, Navier-Stokes equations, Theory and Numerical Analysis, 2nd. ed., North Holland, Amsterdam, 1979.

[18] R. Temam, Infinite Dimensional Dynamical Systems in Mechanics and Physics, Springer, New York, 1988.

[19] R. Temam, Navier-Stokes Equations and Nonlinear Functional Analysis, 2nd Edition, SIAM, Philadelphia, 1995. 